\title{
Pruebas de laboratorio y desenlace clínico en pacientes hospitalizados por la COVID-19
}

\section{Laboratory tests and clinical outcome in hospitalized patients for COVID-19}

\author{
Jaime Antonio Collins-Camones', Fiorella \\ Vanesa Loyola-Salvatierra², Roxana Liliana \\ Gamarra-Lazo ${ }^{3}$, Celia Joanna \\ Páucar-Miranda ${ }^{4}$, Moisés Gonzalo \\ Cárdenas-Ruiz ${ }^{3}$, Nikolai Grande-Castro ${ }^{5}$ \\ Juan Carlos Palacios-Calcina 6 , Patricia Nelly \\ Reyes-Payano ${ }^{3}$,Amanda Beatriz Blanco-Noriega ${ }^{7}$ \\ Gerson Edwin Díaz-Gonzales ${ }^{8}$, Sergio Enrique \\ Alvizuri-Pastor 9
}

Collins-Camones JA, Loyola-Salvatierra FV, Gamarra-Lazo RL, PáucarMiranda CJ, Cárdenas-Ruiz MG, Grande-Castro N, Palacios-Calcina JC, Reyes-Payano PN, Blanco-Noriega AB, Díaz-Gonzales GE,

Alvizuri-Pastor SE. Pruebas de laboratorio y desenlace clínico en pacientes hospitalizados por la COVID-19. 2021;34(2): 53-60. https://doi.org/10.36393/spmi.v34i2.596

\begin{abstract}
RESUMEN
Objetivo: Identificar los resultados de laboratorio asociados con el riesgo de muerte en pacientes hospitalizados por la COVID-19. Material y métodos: estudio de cohorte retrospectivo de pacientes admitidos por la COVID-19 en el Hospital Nacional EsSalud Guillermo Almenara Irigoyen de Lima, entre el 2 I de marzo y el I 2 de mayo 2020. Las pruebas de laboratorio incluidas en el estudio fueron las del ingreso al hospital. Se hizo un análisis de Cox para determinar factores asociados con muerte. Resultados: un total de 433 pacientes fueron incluidos: 253 casos (58\%) con RT-PCR positivo y 180 casos (42\%) con prueba rápida de anticuerpos reactiva. El 55\% (240/433) y el 59\% (256/433) tuvo leucocitosis y linfopenia relativa ( $\leq 10 \%)$, respectivamente. El 56\% (49/87) tuvo dímero-D mayor a dos mg/L. Cifras mayores al normal se presentaron en 39\% (I52/393) para urea y $19 \%$ (77/397) para creatinina, así como en $73 \%(228 / 313)$ para aspartato aminotransferasa y 62\% (193/3I0) para alanina aminotransferasa. De otro lado, 54\% (I80/336) tuvo proteína C reactiva mayor a 150 mg/L, 42\% (I08/258) lactato deshidrogenasa mayor a $450 \mathrm{U} / \mathrm{L}$ y $57 \%$ (52/9l) ferritina mayor a mil ng/mL De forma subsiguiente, leucocitosis (HRa 2,72; IC955; I, I 2 6,60), menos de trescientas mil plaquetas por mm3 (HRa 2,36; IC95\%; I,23-4,52), e incrementos en los valores normales de creatinina (HRa 3,03; IC95\%; I,4I-6,48) como de lactato deshidrogenasa por encima de $450 \mathrm{U} / \mathrm{L}$ (HRa 2,4I; IC95\%; I,29-4,49) se asociaron de forma independiente con muerte. Conclusión: la muerte de los pacientes hospitalizados por la COVID-I 9 fue asociada de forma independiente con leucocitosis, menos de trescientas mil plaquetas por mm3, creatinina por encima del normal y lactato deshidrogenasa mayor a $450 \mathrm{U} / \mathrm{L}$.
\end{abstract}

Palabras claves: COVID-19, hemograma, bioquímica sanguínea, mortalidad.

Médico internista e infectólogo. Servicio de Infectología, Departamento de Medicina Interna, Hospital Nacional EsSalud Guillermo Almenara Irigoyen, Lima, Perú.

2 Médica residente de medicina interna. Servicio de Infectología, Departamento de Medicina Interna, Hospital Nacional EsSalud Guillermo Almenara Irigoyen, Lima, Perú.

3 Médico patólogo clínica. Servicio de Bioquímica, Hospital Nacional EsSalud Guillermo Almenara Irigoyen, Lima, Perú.

4 Médica patóloga clínica. Servicio de Microbiología, Hospital Nacional EsSalud Guillermo Almenara Irigoyen, Lima, Perú.
Médico patólogo clínico. Unidad de Inmuno-diagnóstico Hospital Nacional EsSalud Guillermo Almenara Irigoyen, Lima, Perú. Médico patólogo clínico. Laboratorio de Emergencia, Hospital Nacional EsSalud Guillermo Almenara Irigoyen, Lima, Perú. Licenciada en Tecnología Médica Servicio de Bioquímica, Hospital Nacional EsSalud Guillermo Almenara Irigoyen, Lima, Perú.

\& Médico emergenciólogo. Departamento de Emergencia, Hospital Nacional EsSalud Guillermo Almenara Irigoyen, Lima, Perú.

Médico patólogo clínico. Departamento de Patología Clínica, Hospital Nacional EsSalud Guillermo Almenara Irigoyen, Lima, Perú. 


\section{ABSTRACT}

Objective:To identify laboratory results associated with the risk of death in patients hospitalized for COVID- 19.

Material and methods: retrospective cohort study of patients admitted for COVID-19 at the Hospital Nacional EsSalud Guillermo Almenara Irigoyen in Lima, between March 21 and May 12, 2020. The laboratory tests included in the study were those of hospital admission. A Cox analysis was done to determine factors associated with death.

Results: a total of 433 patients were included: 253 cases (58\%) with positive RT-PCR and 180 cases (42\%) with reactive rapid antibody test. And, 55\% (240/433) and 59\% (256/433) had leukocytosis and relative lymphopenia ( $\leq 10 \%)$, respectively. 56\% (49/87) had D-dimer greater than two $\mathrm{mg} / \mathrm{L}$. However, higher than normal values were presented in $39 \%$ (152/393) for urea and 19\% (77/397) for creatinine, as well as $73 \%(228 / 3 / 3)$ for aspartate aminotransferase and $62 \%$ $(193 / 310)$ for alanine aminotransferase. On the other hand, $54 \%$ (I80/336) had C-reactive protein greater than $150 \mathrm{mg} / \mathrm{L}$, $42 \%$ (108/258) lactate dehydrogenase greater than $450 \mathrm{U} / \mathrm{L}$ and $57 \%(52 / 91)$ ferritin greater than one thousand $\mathrm{ng} / \mathrm{mL}$. Subsequently, leukocytosis (HRa 2.72; IC955; I.I 2-6.60), less than three hundred thousand platelets per mm3 (HRa 2.36; C195\%; 1.23-4.52), and increases in normal creatinine values (HRa 3.03; Cl95\%; I.4 I-6.48) as lactate dehydrogenase above 450 U/L (HRa 2.4I; Cl95\%; I.29-4.49) were independently associated with death.

Conclusion: the death of hospitalized patients by COVID-19 was independently associated with leukocytosis, less than three hundred thousand platelets per $\mathrm{mm} 3$, creatinine above normal and lactate dehydrogenase greater than $450 \mathrm{U} / \mathrm{L}$.

Keywords: COVID-19, blood count, blood biochemistry, mortality.

\section{INTRODUCCIÓN}

La pandemia de la COVID-19, con varios epicentros, sigue vigente en el mundo. Esta ha acarreado cerca de ciento treinta millones de casos confirmados y alrededor de tres millones de muertos. ${ }^{1}$ Las pruebas de laboratorio son una herramienta importante en la evaluación inicial y el seguimiento de pacientes hospitalizados por la COVID-19. Como tal, tempranamente en el curso de la pandemia, las alteraciones en las pruebas de laboratorio fueron caracterizadas. ${ }^{2}$ Adicionalmente, diversos estudios han asociado los resultados de pruebas de laboratorio con la gravedad de la enfermedad. También con el pronóstico o desenlace de los pacientes, aunque en menor número para este caso. ${ }^{3-6}$

En el medio actual, hasta donde se conoce, no se ha reportado la asociación de resultados de pruebas de laboratorio con el desenlace de los pacientes. En tal sentido, la presente investigación se propuso determinar cuál fue el perfil de los resultados de las pruebas de laboratorio al ingreso de pacientes hospitalizados por la COVID-19, y la asociación de dichos resultados con el riesgo de muerte de los pacientes.

\section{MATERIAL Y MÉTODOS}

Se realizó un estudio observacional, longitudinal y retrospectivo en todos los pacientes con evidencia etiológica de la COVID-19 admitidos en la emergencia del Hospital Nacional EsSalud Guillermo Almenara Irigoyen, entre el 21 de marzo y el 12 de mayo de 2020.

Para el estudio, se rastrearon los resultados de las siguientes pruebas de diagnóstico: reacción en cadena de polimerasa con transcripción reversa en tiempo real (RT-PCR) y prueba rápida de anticuerpos para el SARS-CoV-2. La primera fue procesada en el Instituto Nacional de Salud. Del mismo modo, se rastrearon los resultados de las siguientes pruebas de laboratorio como parte de la evaluación de los pacientes al ingreso: leucocitos, linfocitos, hemoglobina y plaquetas; tiempo de protrombina, tiempo parcial de tromboplastina, fibrinógeno y dímero-D; glucemia, urea y creatinina; alanina aminotransferasa (ALAT o TGP), aspartato aminotransferasa (ASAT o TGO), fosfatasa alcalina (FA), gamma glutamil transferasa (GGT) y albúmina; lactato deshidrogenasa $(\mathrm{LDH})$, proteína $\mathrm{C}$ reactiva, ferritina sérica y troponina I.

De este modo, fueron incluidos los pacientes con pruebas de laboratorio realizadas dentro de las 48 horas del ingreso al hospital, así como aquellos que contaron, por lo menos, con un hemograma. El rastreo de las pruebas de laboratorio fue realizado en una base de datos electrónica donde se reportan las pruebas de laboratorio en el hospital. La base de datos se denomina Sistema de Gestión Hospitalaria. Los detalles del procedimiento seguido por el laboratorio se presentan como apéndice del artículo.

Por otra parte, como desenlace clínico de los pacientes, se consideraron las siguientes categorías: fallecidos versus referidos a otros centros para continuar tratamiento o quienes fueron dados de alta al domicilio. Los datos generales de los pacientes y su desenlace clínico fueron rastreados de la historia clínica electrónica del hospital.

Se empleó estadística descriptiva, ji-cuadrado o prueba exacta de Fisher para proporciones y la prueba de t de Student para medias. Para identificar los factores relacionados con el desenlace de muerte, se hizo un análisis bivariado y multivariado con el modelo de riesgos proporcionales de Cox. El análisis multivariado fue ajustado con las variables clínicas que se asociaron de forma independiente con el desenlace de los pacientes en un estudio clínico previo con la misma población de estudio. Estas variables fueron las siguientes: edad mayor de 60 años, obesidad, hipotiroidismo, enfermedad renal crónica en general y enfermedad renal crónica estadio 5 en hemodiálisis. Para el análisis, se hizo uso del programa estadístico SPSS para Windows en su versión 25.

El estudio fue autorizado por las jefaturas del Departamento de Emergencia (de entonces) y del Departamento de Patología Clínica. Aparte, se revisaron fuentes secundarias para los datos clínicos y la fuente primaria para los datos de laboratorio. En ambos casos, la confidencialidad de los datos de los pacientes fue preservada. 


\section{RESULTADOS}

\section{De la población de estudio}

Entre el período de estudio ingresaron al hospital 533 pacientes con evidencia etiológica de la COVID-19. De estos, 100 fueron excluidos del estudio por no contar con pruebas de laboratorio al ingreso, por lo menos hemograma en las primeras 48 horas. De esta forma, la población de estudio fue conformada por 433 casos. La media de edad de los pacientes fue 61 años, con una desviación estándar de 15 años. Por su parte, las mujeres fueron 123 (28\%).

\section{Del diagnóstico}

La evidencia etiológica del SARS-CoV-2 en los 433 pacientes del estudio se obtuvo mediante RT-PCR en 253 casos (58\%) o prueba rápida de anticuerpos en los 180 casos restantes (42\%). Un 10\% (26/253) de quienes tuvieron RT-PCR positivo presentó prueba rápida de anticuerpos no reactivas. Los patrones de anticuerpos de las pruebas rápidas en quienes el diagnóstico de la COVID-19 se hizo solo mediante este recurso diagnóstico fueron los siguientes: IgM e IgG en 107 (59,4\%); IgM en 24 (13,3\%); IgG en 16 (9\%); y no se precisó el tipo de anticuerpo en 33 $(18,3 \%)$.

\section{Análisis de proporciones}

Los resultados del análisis de proporciones se presentan en la Tabla 1. Según estos resultados, los pacientes que fallecieron tuvieron al ingreso proporciones significativamente mayores de incrementos en leucocitos, urea, creatinina, fosfatasa alcalina, proteína $\mathrm{C}$ reactiva, lactato deshidrogenasa y troponina I en comparación con los pacientes referidos o de alta. De modo similar, los pacientes del primer grupo tuvieron al ingreso proporciones significativamente mayores de linfopenia relativa y de hipoalbuminemia en contraste con los pacientes referidos o de alta. Adicionalmente, aunque fue muy poco frecuente, los pacientes que fallecieron tuvieron al ingreso proporciones significativamente mayores de incremento en los tiempos de protrombina y de tromboplastina activado en relación con los pacientes referidos o de alta.

En los casos de linfocitos, dímero-D, glucemia, proteína $\mathrm{C}$ reactiva, lactato deshidrogenasa y ferritina, se consideró un valor aproximado a la media como punto de corte para las categorías de la variable.

\section{Análisis de medias}

Los resultados del análisis de medias se presentan en la Tabla 2. Según estos resultados, los pacientes que fallecieron

Tabla 1. Resultados de las pruebas de laboratorio en proporciones según desenlace

\begin{tabular}{|c|c|c|c|c|}
\hline Variable & n/total [\%] & n/fallecidos (\%) & n/referidos o altas (\%) & P valor \\
\hline Leucocitos $>10000$ por $\mathrm{mm}^{3}$ & $240 / 433[55]$ & $119 / 172(69)$ & $121 / 261(46)$ & 0,000 \\
\hline Linfocitos $<20 \%$ & $368 / 433[85]$ & $158 / 172(92)$ & $210 / 261(80)$ & $0,001^{*}$ \\
\hline Linfocitos $<10 \%$ & $256 / 433$ [59] & $126 / 172(73)$ & $130 / 261(50)$ & 0,000 \\
\hline Hemoglobina $>14 \mathrm{~g} / \mathrm{dL}$ & $181 / 432[42]$ & $65 / 172(38)$ & $116 / 260(45)$ & 0,170 \\
\hline Plaquetas & & & & $0,122^{*}$ \\
\hline$<150000$ por $\mathrm{mm}^{3}$ & $15 / 433(3,5)$ & $9 / 172(5,2)$ & $6 / 261(2,3)$ & \\
\hline$>450000$ por $\mathrm{mm}^{3}$ & $41 / 433(9,5)$ & $12 / 172(6,9)$ & $29 / 261(11)$ & \\
\hline $\mathrm{TP}>14 \mathrm{seg}$ & $13 / 282(4,6)$ & $10 / 120(8,3)$ & $3 / 162(1,9)$ & $0,018^{*}$ \\
\hline $\mathrm{TTP}>45 \mathrm{seg}$ & $17 / 282(6)$ & $13 / 120(11)$ & $4 / 162(2,5)$ & $0,005^{*}$ \\
\hline Fibrinógeno $>400 \mathrm{mg} / \mathrm{dL}$ & $247 / 281(88)$ & $108 / 119(91)$ & $139 / 162(86)$ & $0,267^{*}$ \\
\hline Dímero-D > 2 mg/L & $49 / 87(56)$ & $23 / 39(59)$ & $26 / 48(54)$ & 0,653 \\
\hline Glucemia $>150 \mathrm{mg} / \mathrm{dL}$ & $125 / 394(32)$ & $54 / 159(34)$ & $71 / 235(30)$ & 0,433 \\
\hline Urea $>45 \mathrm{mg} / \mathrm{dL}$ & $152 / 393(39)$ & $80 / 157(51)$ & $72 / 236(30)$ & 0,000 \\
\hline Creatinina $>1,1 \mathrm{mg} / \mathrm{dL}$ & 77 / 397 (19) & $46 / 160(29)$ & $31 / 237(13)$ & 0,000 \\
\hline ASAT o $\mathrm{TGO}>40 \mathrm{U} / \mathrm{L}$ & $228 / 313(73)$ & $101 / 131(77)$ & $127 / 182(70)$ & 0,151 \\
\hline ALAT o TGP > $40 \mathrm{U} / \mathrm{L}$ & $193 / 310(62)$ & 79 / $128(62)$ & $114 / 182(63)$ & 0,870 \\
\hline Fosfatasa alcalina $>129 \mathrm{U} / \mathrm{L}$ & $165 / 309(53)$ & $77 / 128(60)$ & 88 / $181(49)$ & 0,045 \\
\hline $\mathrm{GGT}>73 \mathrm{U} / \mathrm{L}$ & $214 / 311(69)$ & $90 / 129(70)$ & $124 / 182(68)$ & 0,759 \\
\hline Albúmina $<3,2 \mathrm{~g} / \mathrm{dL}$ & $109 / 309(35)$ & 62 / $127(49)$ & $47 / 182(26)$ & 0,000 \\
\hline Proteína $\mathrm{C}$ reactiva $>150 \mathrm{mg} / \mathrm{L}$ & $180 / 336(54)$ & $88 / 141(62)$ & $92 / 195(47)$ & 0,006 \\
\hline $\mathrm{LDH}$ o $\mathrm{DHL}>450 \mathrm{U} / \mathrm{L}$ & $108 / 258(42)$ & $66 / 102(65)$ & $44 / 156(28)$ & 0,000 \\
\hline Ferritina $>1000 \mathrm{ng} / \mathrm{mL}$ & $52 / 91(57)$ & $26 / 41(63)$ & $26 / 50(52)$ & $0,295^{*}$ \\
\hline Troponina $\mathrm{I}>0,06 \mathrm{ng} / \mathrm{mL}$ & $13 / 48(27)$ & $11 / 20(55)$ & $2 / 28(7)$ & $0,000^{*}$ \\
\hline
\end{tabular}

*Prueba exacta de Fisher 
Tabla 2. Resultados de las pruebas de laboratorio en medias según desenlace.

\begin{tabular}{|c|c|c|c|c|}
\hline Variable (desviación estándar; n) & total $(\mathrm{DE} ; \mathbf{n})$ & Fallecidos (DE; n) & Referidos o altas (DE; n) & P valor \\
\hline Leucocitos $\times 10^{3}$ por $\mathrm{mm}^{3}$ & $11,7[5,3 ; 433]$ & $13,5(5,6 ; 172)$ & $10,4(4,7 ; 261)$ & 0,000 \\
\hline Linfocitos en proporción & $11,4(7,6 ; 433)$ & $9,1(6 ; 172)$ & $13(8 ; 261)$ & 0,000 \\
\hline Hemoglobina, g/dL & $13,6(1,8 ; 432)$ & $13,4(1,8 ; 172)$ & $13,8(1,8 ; 260)$ & 0,056 \\
\hline Plaquetas $\mathrm{x} 10^{3}$ por $\mathrm{mm}^{3}$ & $314(107 ; 433)$ & $292(104 ; 172)$ & $328(107 ; 261)$ & 0,001 \\
\hline Tiempo de protrombina, seg & $11,8(6,8 ; 282)$ & $12,8(10,2 ; 120)$ & $11,1(1,3 ; 162)$ & 0,046 \\
\hline Tiempo de tromboplastina, seg & $34,2(12,3 ; 282)$ & $36,4(17,6 ; 120)$ & $32,4(5,4 ; 162)$ & 0,006 \\
\hline Fibrinógeno, mg/dL & $4,6(0,4 ; 281)$ & $4,7(0,3 ; 119)$ & $4,6(0,4 ; 162)$ & 0,216 \\
\hline Dímero-D, mg/L & $2,6(1,6 ; 87)$ & $2,8(1,5 ; 39)$ & $2,5(1,7 ; 48)$ & 0,477 \\
\hline Glucemia, mg/dL & $152(79 ; 394)$ & $161(93 ; 159)$ & $146(67 ; 235)$ & 0,053 \\
\hline Urea, mg/dL & $53(47 ; 393)$ & $64(59 ; 157)$ & $45(35 ; 236)$ & 0,000 \\
\hline Creatinina, mg/dL & $1,4(2,5 ; 397)$ & $1,6(2,6 ; 160)$ & $1,3(2,4 ; 237)$ & 0,175 \\
\hline ASAT o TGO, U/L & $69(52 ; 313)$ & $78(64 ; 131)$ & $62(41 ; 182)$ & 0,007 \\
\hline ALAT o TGP, U/L & $68(63 ; 310)$ & $66(59 ; 128)$ & $70(66 ; 182)$ & 0,625 \\
\hline Fosfatasa alcalina, U/L & $158(87 ; 309)$ & $169(85 ; 128)$ & $150(87 ; 181)$ & 0,063 \\
\hline GGT, U/L & $170(1750 ; 311)$ & $170(185 ; 129)$ & $170(168 ; 182)$ & 0,978 \\
\hline Albúmina, g/dL & $3,4(0,4 ; 309)$ & $3,2(0,4 ; 127)$ & $3,5(0,4 ; 182)$ & 0,000 \\
\hline Proteína $\mathrm{C}$ reactiva, $\mathrm{mg} / \mathrm{L}$ & $173(106 ; 336)$ & $192(106 ; 141)$ & $159(104 ; 195)$ & 0,005 \\
\hline LDH o DHL, U/L & $477(243 ; 258)$ & $596(269 ; 102)$ & $399(187 ; 156)$ & 0,000 \\
\hline Ferritina, ng/mL & $1149(598 ; 91)$ & $1307(625 ; 41)$ & $1019(548 ; 50)$ & 0,021 \\
\hline Troponina I, ng/mL & $0,25(0,93 ; 48)$ & $0,54(1,4 ; 20)$ & $0,05(0,1 ; 28)$ & 0,067 \\
\hline
\end{tabular}

Tabla 3. Análisis bivariado y multivariado según desenlace

\begin{tabular}{|c|c|c|}
\hline Variables & $\begin{array}{l}\text { Análisis bivariado } \\
\text { HRc [IC95\%] }\end{array}$ & $\begin{array}{l}\text { Análisis multivariado } \\
\text { HRa [IC95\%] }\end{array}$ \\
\hline Leucocitos $>10 \times 10^{3}$ por $\mathrm{mm}^{3}$ & $1,89[1,36-2,61]^{* * *}$ & $2,72[1,12-6,60]^{* *}$ \\
\hline Linfocitos $<10 \%$ & $1,66[1,19-2,34]^{* *}$ & $1,86[0,88-3,91]$ \\
\hline Plaquetas $<300 \times 10^{3}$ por $\mathrm{mm}^{3}$ & $1,49[1,10-2,03]^{* *}$ & $2,36\left[1,23-4,52^{*}\right.$ \\
\hline Glucemia $>150 \mathrm{mg} / \mathrm{dL}$ & $1,08[0,78-1,51]$ & $0,55[0,31-0,97]^{*}$ \\
\hline Urea $>45 \mathrm{mg} / \mathrm{dL}$ & $1,61[1,18-2,20]^{* *}$ & $1,42[0,76-2,66]$ \\
\hline Creatinina $>1,1 \mathrm{mg} / \mathrm{dL}$ & $1,78[1,26-2,52]^{* *}$ & $3,03[1,41-6,48]^{* *}$ \\
\hline ASAT o TGO $>40 \mathrm{U} / \mathrm{L}$ & $1,31[0,87-1,98]$ & $0,93[0,44-1,99]$ \\
\hline ALAT o TGP $>40 \mathrm{U} / \mathrm{L}$ & $1,00[0,69-1,43]$ & $1,44[0,68-3,08]$ \\
\hline Fosfatasa alcalina $>129 \mathrm{U} / \mathrm{L}$ & $1,56[1,09-2,23]^{*}$ & $1,59[0,86-2,96]$ \\
\hline $\mathrm{GGT}>73 \mathrm{U} / \mathrm{L}$ & $0,99[0,68-1,45]$ & $0,97[0,47-1,96]$ \\
\hline Albúmina $<3,2 \mathrm{~g} / \mathrm{dL}$ & $1,57[1,11-2,22]^{*}$ & $0,84[0,47-1,52]$ \\
\hline Proteína $\mathrm{C}$ reactiva $>150 \mathrm{mg} / \mathrm{L}$ & $1,55[1,10-2,19]^{*}$ & $1,08[0,59-1,98]$ \\
\hline LDH o DHL > $450 \mathrm{U} / \mathrm{L}$ & $2,74[1,82-4,14]^{* * *}$ & $2,41[1,29-4,49]^{* *}$ \\
\hline
\end{tabular}


tuvieron al ingreso un nivel medio significativamente mayor en leucocitos, tiempos de protrombina y de tromboplastina activado, urea, aspartato aminotransferasa, proteína $\mathrm{C}$ reactiva, lactato deshidrogenasa y ferritina en comparación con los pacientes referidos o de alta. De modo similar, los pacientes del primer grupo tuvieron al ingreso niveles medios significativamente más bajos de linfopenia relativa y de albúmina en sangre en contraste con los pacientes referidos o de alta.

\section{Análisis de Cox}

Los resultados del análisis de Cox se presentan en la Tabla 3. Por su número reducido de casos, ferritina y troponina I no fueron incluidos en el análisis. Tampoco hemoglobina por el resultado del análisis bivariado y por su poca relevancia clínica en relación al riesgo de muerte hasta donde se conoce. El Hazard ratio crudo (HRc) para hemoglobina mayor versus menor a $13 \mathrm{gr} / \mathrm{dL}$ fue 1,19 (IC95\%; 0,87-1,63; p=0,268). Entretanto, los tiempos de protrombina y de tromboplastina activado no formaron parte del modelo final, aun cuando los resultados del análisis bivariado fueron significativos, ya que los casos evaluables para el análisis multivariado se reducirían de 163 a 128 casos. Adicionalmente, porque la inclusión de estas pruebas no produjo mejoras en el modelo. El HRc para tiempo de protrombina mayor versus menor a 14 segundos fue 12,13 (IC95\%; 1,11-4,09; p=0,022), mientras que el HRc para tiempo de tromboplastina activado mayor versus menor a 45 segundos fue 1,96 (IC95\%; 1,09-3,51; $\mathrm{p}=0,023)$. Según el análisis multivariado presentado, ajustado por edad mayor a 60 años, obesidad, enfermedad renal crónica en general y enfermedad renal crónica estadio 5 en hemodiálisis regular, tanto leucocitosis como un conteo de plaquetas menor a trescientos mil por $\mathrm{mm}^{3}$, así como un incremento por encima del normal de creatinina y de lactato deshidrogenasa por encima de $450 \mathrm{U} / \mathrm{L}$, se asociaron de forma independiente con el riesgo de muerte de los pacientes. No hubo casos de hipotiroidismo cuando participaron del análisis multivariado. Por otra parte, en el análisis multivariado, la glucemia mayor a $150 \mathrm{mg} / \mathrm{dL}$ se comportó como factor protector.

\section{DISCUSIÓN}

El estudio propuesto es el primero a nivel nacional que asocia resultados de pruebas de laboratorio con el riesgo de muerte de pacientes hospitalizados por la COVID-19 en un establecimiento de salud de nivel III- $2^{7}$. En este sentido, la muerte de los pacientes fue asociada de forma independiente con leucocitosis, conteo menor de plaquetas, e incrementos en los valores tanto de creatinina como de lactato deshidrogenasa en sangre.

Las pruebas de laboratorio son una herramienta importante en la evaluación inicial y el seguimiento de pacientes hospitalizados por la COVID-19. La afección sistémica por parte del SARS-CoV-2 produce daño tisular y funcional de diversos órganos. Estos cambios pueden medirse mediante pruebas de laboratorio comunes. Del mismo modo, la intensidad de la respuesta inflamatoria del organismo puede ser medida a través de este tipo de pruebas, como la proteína $\mathrm{C}$ reactiva y la ferritina, aunque también con pruebas menos comunes, por ejemplo, la interleucina- 6 .

Desde otro punto de vista, la evaluación de las tendencias en las alteraciones de las pruebas de laboratorio es un complemento adecuado para determinar, junto con el cuadro clínico, el pronóstico o desenlace de los pacientes. La frecuencia con la que se deberían realizar las pruebas de laboratorio dependerá de la gravedad de la enfermedad. En general, durante la primera semana de hospitalización de un paciente no crítico se debería contar, por lo menos, con tres perfiles de laboratorio, incluido el del ingreso. En este sentido, cada establecimiento de salud debería definir los perfiles de laboratorio con los que se evaluarán de forma rutinaria a los pacientes hospitalizados, así como también debería establecer un mecanismo de supervisión u organización para que se cumpla de forma homogénea.

Comparar los resultados de las pruebas de laboratorio con el de otras cohortes hospitalarias grandes y similares a la presentada es complicado, puesto que los reportes no son homogéneos. Varían los puntos de corte de normalidad y sobre todo los rangos de las categorías de las variables. No obstante, la alteración más resaltante y constante fue la respuesta de leucocitos. Mientras que la mayoría de los pacientes evaluados tuvo leucocitosis al ingreso, menos de la quinta parte de los pacientes de otras regiones tuvo tal alteración: $6 \%^{8}, 10 \%{ }^{9}$ y $12 \%{ }^{10}$ en China; $13 \%$ en Nueva York, EE. UU. ${ }^{11}$; y $17 \%$ en Londres, Reino Unido ${ }^{12}$. Una diferencia similar fue observada en los porcentajes de leucopenia. Solo 1,4\% (6/433) de los pacientes en la presente cohorte tuvo leucopenia, mientras que en China fue $19 \%{ }^{9}$ y $34 \%^{8}$; en Nueva York, $16 \%{ }^{11}$; y en Londres, $9 \%{ }^{12}$. Estas variaciones pueden ser expresión de diferencias regionales, considerando que un estudio local ${ }^{13}$ tuvo hallazgos consistentes con la presente investigación, aunque con una proporción de leucocitosis mayor (55\% vs. $62 \%)$ y una mediana de linfopenia relativa más profunda (11\% vs. $8 \%)$. Una hipótesis para explicar estas diferencias regionales puede plantear que la leucocitosis en la admisión de los pacientes es una manifestación tardía de la enfermedad en el medio actual. Sin embargo, no habría sustento para ello, ya que la mediana del tiempo de enfermedad en el estudio local indicado fue de siete días. Esta alteración podría estar relacionada, más bien, con la gravedad de los pacientes cuando fueron admitidos.

En cuanto a resultados de otras pruebas de laboratorio, la presente cohorte fue más afectada en comparación a otras similares. Por ejemplo, se tuvo más casos con lo siguiente: dímero-D mayor a 2,0 mg/L que en Londres ${ }^{12}$ (56\% vs. $30 \%$ ); aspartato aminotransferasa mayor a $40 \mathrm{U} / \mathrm{L}$ que en China ( $73 \%$ vs. $22 \%{ }^{8}, 28 \%{ }^{9}$ y $30 \%{ }^{10}$, respectivamente) y Nueva York $^{11}$ (73\% vs. 47\%); creatinina sérica mayor a $1,1 \mathrm{mg} / \mathrm{dl}$ que en Nueva York ${ }^{11}$ y Londres ${ }^{12}$ (33\% vs. $15 \%$ [>1,5 mg/dl] y $26 \%$ [>1,25 mg/dl], respectivamente); proteína $\mathrm{C}$ reactiva mayor a $150 \mathrm{mg} / \mathrm{L}$ que en $\mathrm{China}^{10}$ y 
Londres $^{12}$ (54\% vs. $26 \%$ y $52 \%$ [>100 mg/L en ambos casos], respectivamente); lactato deshidrogenasa mayor a 450 U/L que en China $^{10}$ (42\% vs. $\left.25 \%\right)$; ferritina mayor a mil ng/lL que en Londres ${ }^{12}$ (57\% vs. 41\%). En contraste, la media de plaquetas de los pacientes del centro evaluado fue superior al de dos estudios en China (314 mil vs. 168 mil $^{8}$ y 184 mil $^{9}$, respectivamente). De modo similar, el porcentaje de hipoalbuminemia fue menor que en China ${ }^{10}$ y Londres ${ }^{12}$ (35\% vs. 59\% y 75\% [ $<3,5$ gr/dl en ambos casos], respectivamente).

Por otra parte, al interior de la presente cohorte, queda claro, tanto por el análisis de proporciones como de medias, que hay diferencias significativas en los resultados de las pruebas de laboratorio al ingreso entre quienes mueren o sobreviven a la COVID-19, en concordancia con otros estudios. ${ }^{12-18}$ No obstante, la investigación presentada fue aún más lejos. Con base en el análisis de Cox, cuatro pruebas de laboratorio se asociaron de forma independiente con la muerte de los pacientes. En primer lugar, los pacientes con leucocitosis (es decir, un conteo de leucocitos mayor a diez mil por $\mathrm{mm}^{3}$ ) presentaron cerca de tres veces más riesgo de morir que los pacientes con un conteo de leucocitos normal o bajo al ingreso, consistente con otros reportes. ${ }^{10,12,16}$ En segundo lugar, los pacientes con un conteo de plaquetas por debajo de trescientos mil por $\mathrm{mm}^{3}$ presentaron dos veces más riesgo de morir que los pacientes con un conteo de plaquetas por encima de dicha cifra, lo que también fue concordante con otros reportes. ${ }^{12,15,19} \mathrm{En}$ tercer lugar, los pacientes con una creatinina sérica superior al normal presentaron tres veces más riesgo de morir que los pacientes con una creatinina normal al ingreso, en línea con otros reportes. ${ }^{20,21} \mathrm{Al}$ respecto, es importante hacer notar que una quinta parte de los pacientes de la cohorte entera presentaron una creatinina sérica elevada, porción tres veces superior a la del siete por ciento de pacientes de la cohorte con historia de enfermedad renal crónica, incluido los pacientes que estaban en hemodiálisis regular. Esto quiere decir que el exceso puede ser atribuible en gran medida a la COVID-19 como injuria renal. En cuarto lugar, los pacientes con lactato deshidrogenasa mayor a $450 \mathrm{U} / \mathrm{L}$ presentaron dos veces más riesgo de morir que los pacientes con un lactato deshidrogenasa por debajo de dicha cifra, consistente también con otros estudios. . $^{9} 10,15,22$

Resulta interesante, aunque no extraño, que el lactato deshidrogenasa se comporte como factor de riesgo independiente de muerte. Su alteración expresa daño tisular difuso o de varios órganos en el contexto de la COVID-19 como enfermedad sistémica. De modo similar, se puede interpretar el hecho de que quienes fallecieran presentaran un nivel medio de aspartato aminotransferasa más alto al ingreso en comparación con aquellos que fueron referidos o dados de alta. En esa misma línea, la injuria renal también adquiere relevancia en el pronóstico de los pacientes afectados.

Por otra parte, resulta interesante que la glucemia mayor a $150 \mathrm{mg} / \mathrm{dl}$ al ingreso se comporte como factor protector, según el análisis multivariado. Al respecto, la prevalencia de diabetes mellitus en la cohorte original de 533 pacientes fue $22 \%$ (dato no mostrado), lo cual puede explicar en gran medida el nivel de glucemia al ingreso en la cohorte de la presente investigación.

Otras pruebas de laboratorio han sido reportadas como factores de riesgo independiente de muerte en otros estudios. Tales son los casos de creatina-fosfocinasa ${ }^{9,17}$, hipo-albuminemia ${ }^{12}$, proteína $\mathrm{C}$ reactiva ${ }^{17,23}$, dímero- $\mathrm{D}^{18}$, ${ }^{24}$, injuria hepática con colestasis y patrón mixto ${ }^{25}$, o simplemente un incremento de aspartato aminotransferasa, aunque no sea específica del hígado ${ }^{14,21,26}$, y troponina $\mathrm{I}^{27}$ En estas investigaciones, la determinación del riesgo fue similar al del presente estudio, con el Hazard ratio ajustado (HRa) como medida del riesgo independiente de muerte.

Merecen una mención especial dos pruebas que podrían formar parte de los análisis de laboratorio en la admisión de los pacientes hospitalizados por la COVID-19. Estas son la hormona estimulante del tiroides (TSH, por sus siglas en inglés) y la procalcitonina. Respecto a la TSH, esta prueba puede ser útil dado el alto porcentaje de tirotoxicosis reportado como problema incidente en el norte de Italia $(20 \%, 58 / 287)^{28}$, lo cual puede ser explicado una vez más por el hecho de la COVID-19 como enfermedad sistémica. Por otra parte, la prueba de procalcitonina es útil para discernir una coinfección bacteriana al ingreso o en el curso de la hospitalización por la COVID-19, y racionalizar el uso de antibacterianos. De hecho, la prueba puede ser positiva $(>0,5 \mathrm{ng} / \mathrm{ml})$ entre un seis a $17 \%$ de pacientes admitidos por la COVID-19 en hospitalización de urgencias. ${ }^{8,10,11}$ Junto con esto, se encuentra el hecho de la leucocitosis como presentación en la admisión de la mayoría de los pacientes evaluados, y que esta también se comporta como factor de riesgo independiente de muerte ${ }^{26}$, por lo que la realización de la prueba estaría justificada en los pacientes con leucocitosis aun si no hubiese manifestación clínica relacionada con la sospecha de coinfección bacteriana. El flujo o toma de las muestras se puede coordinar con el laboratorio para hacer más eficiente el proceso. Por ejemplo, la muestra para procalcitonina puede estar considerada en el perfil de laboratorio inicial y se puede realizar si el laboratorio reporta leucocitosis, para evitar que nuevamente se tome la muestra a los pacientes con todo lo que ello implica en recursos materiales, personal y tiempo.

La limitación principal del estudio fue la falta de uniformidad de las pruebas de laboratorio con la que se contaron en los pacientes. Esto fue reflejo de la naciente organización de la respuesta médica frente a la pandemia de la COVID-19 en los primeros dos meses de la misma. Sin embargo, la falta de uniformidad en la solicitud de las pruebas de laboratorio aún persiste (y debería mejorar), a pesar de que ya están establecidos los perfiles de laboratorio de inicio y de seguimiento. Con esta limitación, el análisis multivariado solo se puedo realizar con menos de la mitad de los casos incluidos en el estudio. No obstante, el tamaño de la población evaluada y los hallazgos son importantes, 
los mismos que contribuirán a mejorar el abordaje clínico de los pacientes afectados por la COVID-19.

En conclusión, durante los primeros dos meses de la pandemia de la COVID-19 en el Hospital Almenara, la mayoría de los pacientes en la admisión presentaron leucocitosis, linfopenia relativa marcada, dímero-D mayor a dos $\mathrm{mg} / \mathrm{L}$, incrementos de las transaminasas por encima del normal, valores de proteína $C$ reactiva mayores a 150 $\mathrm{mg} / \mathrm{L}$ y de ferritina mayores a mil $\mathrm{ng} / \mathrm{ml}$. Por otra parte, las alteraciones en las pruebas de laboratorio al ingreso de los pacientes revelan, fuera del compromiso pulmonar, una mayor o más amplia injuria hepática, renal y cardíaca, así como una respuesta inflamatoria más intensa entre quienes fallecieron respecto de quienes fueron referidos o dados de alta. Finalmente, en un modelo ajustado por edad, obesidad y enfermedad renal crónica tanto general como en hemodiálisis regular, la muerte de los pacientes hospitalizados por la COVID-19 fue asociada de forma independiente con leucocitosis, menos de trescientas mil plaquetas por $\mathrm{mm}^{3}$, e incrementos en los valores normales de creatinina como de lactato deshidrogenasa por encima de $450 \mathrm{U} / \mathrm{L}$.

Los hallazgos del presente estudio dejan abierta la posibilidad para mejorar la respuesta médica del Hospital Almenara frente a la amenaza de la COVID-19. Esto pasa por un trabajo coordinado dentro del área clínica y de esta con el laboratorio.

\section{REFERENCIAS BIBLIOGRÁFICAS}

I. WHO Coronavirus Disease (COVID-19) Dashboard. [Internet]. [Consulted Apr 2, 202I]. Available from: https://covid I9.who.int/ table

2. Zhang ZL, Hou YL, Li DT, Li FZ. Laboratory findings of COVID- I9: a systematic review and meta-analysis. Scand J Clin Lab Invest. 2020 Oct;80(6):44I-447. doi: 10.1080/003655I3.2020.1768587. Epub 2020 May 23.

3. Velavan TP, Meyer CG. Mild versus severe COVID-19: Laboratory markers. Int J Infect Dis. 2020 Jun;95:304-307. doi: 10.1016/j. ijid.2020.04.06I. Epub 2020 Apr 25.

4. Henry BM, de Oliveira MHS, Benoit S, Plebani M,Lippi G. Hematologic, biochemical and immune biomarker abnormalities associated with severe illness and mortality in coronavirus disease 2019 (COVID-19): a meta-analysis. Clin Chem Lab Med. 2020 Jun 25;58(7):I021-1028. doi: 10.15 | $5 / \mathrm{cclm}-2020-0369$.

5. Shang W, Dong J, Ren Y, Tian M, Li W, Hu J, Li Y.The value of clinical parameters in predicting the severity of COVID-19.J MedVirol. 2020 Oct;92(I0):2188-2192. doi: 10.1002/jmv.2603।. Epub 2020 Jun 2.

6. Setiati S, Harimurti K, Safitri ED, Ranakusuma RW, Saldi SRF, Azwar $M K$, et al. Risk factors and laboratory test results associated with severe illness and mortality in COVID-19 patients: A systematic review. Acta Med Indones. 2020 Jul;52(3):227-245.

7. Dirección de Redes Integradas de Salud Lima Centro - Minsa Resolución N I29-2019-DMG-DIRIS-LC. [Internet]. [Consultado 2 abr, 202I]. URL disponible en: http://www.essalud.gob.pe/ hospital-almenara-de-essalud-obtiene-maxima-categoria-por-sualta-especialidad-y-capacidad-resolutiva-en-salud/

8. Guan WJ, Ni ZY, Hu Y, Liang WH, Ou Ch, He JX, et al. Clinical Characteristics of Coronavirus Disease 2019 in China. N Engl J Med. 2020;382(I8): I 708-I720. doi: I0.I056/NEJMoa2002032.

9. Feng Y, Ling Y, Bai T, Xie Y, Huang J, Li J, et al. COVID- I 9 with Different Severities:A Multicenter Study of Clinical Features. Am J Respir Crit Care Med. 2020;20I(II):I380-I388. doi:I0.II64/rccm.202002$04450 \mathrm{C}$
10. Li X, Xu S,Yu M,Wang K, TaoY, Zhou Y, et al. Risk factors for severity and mortality in adult COVID-19 inpatients in Wuhan. J Allergy Clin Immunol.2020; I46(I): I I0-I I8. doi: I0. I0 I6/j.jaci.2020.04.006 (Pend)

II. Goyal P, Choi J, Pinheiro L, Schenck E, Chen R, Jabri A, et al. Clinical Characteristics of Covid- 19 in New York City. N Engl J Med. 2020;382(24):2372-2374. doi: I0.1056/NEJMc20104I9

12. Perez-Guzman PN, Daunt A, Mukherjee S, Crook P, Forlano R, Kont MD, et al. Clinical characteristics and predictors of outcomes of hospitalized patients with COVID-19 in a multi-ethnic London NHS Trust: a retrospective cohort study. Clin Infect Dis. 2020 Aug 7:ciaa I09I. doi: 10.1093/cid/ciaal091.

13. Mejía F, Medina C, Cornejo E, Morello E, Vásquez S, Alave J, et al. Oxygen saturation as a predictor of mortality in hospitalized adult patients with COVID-19 in a public hospital in Lima, Peru. PLoS One. 2020 Dec 28; I5(I2):e0244I7I. doi: I0. I37I/journal.pone.0244I7I.

14. Ding ZY, Li GX, Chen L, Shu C, Song J,Wang W, et al. Association of liver abnormalities with in-hospital mortality in patients with COVID-19. J Hepatol. 2020 Dec 19:SOI68-8278(20)33885-X. doi: 10.1016/j.jhep.2020.12.012. Epub ahead of print.

I5. van Halem K, Bruyndonckx R, van der Hilst J, Cox J, Driesen P, Opsomer M, et al. Risk factors for mortality in hospitalized patients with COVID-19 at the start of the pandemic in Belgium: a retrospective cohort study. BMC Infect Dis. 2020 Nov 27;20(I):897. doi: I0.I | 86/s I 2879-020-05605-3. Erratum in: BMC Infect Dis. 2020 Dec 14;20(I):956

16. Xu PP, Tian RH, Luo S, Zu ZY, Fan B, Wang XM, et al. Risk factors for adverse clinical outcomes with COVID-19 in China: a multicenter, retrospective, observational study. Theranostics. 2020 May I5; I0(14):6372-6383. doi: 10.7150/thno.46833.

17. Giacomelli A, Ridolfo AL, Milazzo L, Oreni L, Bernacchia D, Siano M, et al. 30-day mortality in patients hospitalized with COVID-19 during the first wave of the Italian epidemic: A prospective cohort study. Pharmacol Res. 2020 Aug; I58:10493I. doi: 10.1016/j. phrs.2020.I0493I. Epub 2020 May 22.

18. Zhou F, Yu T, Du R, Fan G, Liu Y, Liu Z, et al. Clinical course and risk factors for mortality of adult inpatients with COVID-19 in Wuhan, China: a retrospective cohort study. Lancet. 2020 Mar 28;395(I0229):I054-I062. doi: I0.10I6/S0I40-6736(20)30566-3. Epub 2020 Mar II.

19. Liu Y, Sun W, Guo Y, Chen L, Zhang L, Zhao S, et al. Association between platelet parameters and mortality in coronavirus disease 2019: Retrospective cohort study. Platelets. 2020 May I8;3 I (4):490496. doi: 10.1080/09537/04.2020.1754383. Epub 2020 Apr 16

20. Russo E, Esposito P, Taramasso L, Magnasco L, Saio M, Briano F, et al. Kidney disease and all-cause mortality in patients with COVID- I 9 hospitalized in Genoa, Northern Italy.J Nephrol.202I Feb;34(I): I73I83. doi: I0.1007/s40620-020-00875-I. Epub 2020 Oct 6.

2I. loannou GN, Locke E, Green P, Berry K, O'Hare AM, Shah JA, et al. Risk Factors for Hospitalization, Mechanical Ventilation, or Death Among 10131 US Veterans With SARS-CoV-2 Infection. JAMA Netw Open. 2020 Sep I;3(9):e20223I0. doi: 10.100I/ jamanetworkopen.2020.22310.

22. Li C, Ye J, Chen Q, Hu W, Wang L, Fan Y, et al. Elevated Lactate Dehydrogenase (LDH) level as an independent risk factor for the severity and mortality of COVID-19. Aging (Albany NY). 2020 Aug I4; I (I5): I5670-I568I. doi: I0. 18632/aging. I03770. Epub 2020 Aug 14.

23. Zhang JJ, Cao YY, Tan G, Dong X, Wang BC, Lin J, et al. Clinical, radiological, and laboratory characteristics and risk factors for severity and mortality of 289 hospitalized COVID-19 patients. Allergy. 202 I Feb;76(2):533-550. doi: I0. I I I I/all.I4496. Epub 2020 Aug 24.

24. Zhang L, Yan X, Fan Q, Liu H, Liu X, Liu Z, Zhang Z. D-dimer levels on admission to predict in-hospital mortality in patients with Covid- 19. JThromb Haemost. 2020 Jun; I 8(6): I 324- I 329. doi: I0. I I I I/ jth. 14859.

25. Chu H, Bai T, Chen L, Hu L, Xiao L, Yao L, et al. Multicenter Analysis of Liver Injury Patterns and Mortality in COVID-19. Front Med (Lausanne). 2020 Oct 20;7:584342. doi: I0.3389/fmed.2020.584342.

26. Chen R, Liang W, Jiang M, Guan W, Zhan C,Wang T, et al. Risk factors 
of fatal outcome in hospitalized subjects with coronavirus disease 2019 From a Nationwide Analysis in China. Chest. 2020 Jul; I 58(I):97105. doi: I0.1016/j.chest.2020.04.010. Epub 2020 Apr I5.

27. Lombardi CM, Carubelli V, lorio A, Inciardi RM, Bellasi A, Canale C, et al. Association of troponin levels with mortality in italian patients hospitalized with coronavirus disease 2019: Results of a Multicenter Study. JAMA Cardiol. 2020 Nov I;5( I I): I274- I280. doi: I0.100 I/jamacardio.2020.3538.
28. Lania A, Sandri MT, Cellini M, Mirani M, Lavezzi E, Mazziotti G. Thyrotoxicosis in patients with COVID-19: the THYRCOV study. Eur J Endocrinol. 2020 Oct; |83(4):38I-387. doi: I0. I530/EJE-20-0335.

\section{CORRESPONDENCIA:}

Jaime Antonio Collins Camones collinslp@gmail.com

Fecha de recepción: 12-04-2021.

Fecha de aceptación: 04-06-2021.

Financiamiento: por los autores.

Conflicto de interés: ninguno, según los autores. 